\title{
Use of Varying Constraints in Optimal 3-D Graph Search for Segmentation of Macular Optical Coherence Tomography Images
}

\author{
Mona Haeker ${ }^{1,2}$, Michael D. Abràmoff ${ }^{1,3}$, Xiaodong $\mathrm{Wu}^{1}$, \\ Randy Kardon ${ }^{3}$, and Milan Sonka ${ }^{1,3}$ \\ Departments of ${ }^{1}$ Electrical \& Computer Engineering, \\ ${ }^{2}$ Biomedical Engineering, and \\ ${ }^{3}$ Ophthalmology \& Visual Sciences, The University of Iowa, \\ Iowa City, IA 52242, USA \\ \{mona-haeker, milan-sonka\}@uiowa.edu
}

\begin{abstract}
An optimal 3-D graph search approach designed for simultaneous multiple surface detection is extended to allow for varying smoothness and surface interaction constraints instead of the traditionally used constant constraints. We apply the method to the intraretinal layer segmentation of 24 3-D optical coherence tomography (OCT) images, learning the constraints from examples in a leave-one-subject-out fashion. Introducing the varying constraints decreased the mean unsigned border positioning errors (mean error of $7.3 \pm 3.7 \mu \mathrm{m}$ using varying constraints compared to $8.3 \pm 4.9 \mu \mathrm{m}$ using constant constraints and $8.2 \pm 3.5 \mu \mathrm{m}$ for the inter-observer variability).
\end{abstract}

\section{Introduction}

Optical coherence tomography (OCT) is becoming an increasingly important modality for the noninvasive assessment of a variety of ocular diseases such as glaucoma, macular edema, and macular degeneration. For assessment of the macular region of the retina, one common scanning protocol involves the acquisition of six linear radial scans in a spoke pattern centered at the fovea (Fig. 11). Although the intraretinal layers are visible on such images, commercially-available quantitative assessment of such scans is currently limited to providing thickness measurements for one layer (e.g., the total retinal thickness on macular scans). Because the intraretinal layers may be affected differently by disease, an intraretinal segmentation approach is needed to enable quantification of individual layer properties, such as thickness or texture. While a few reported approaches for macular intraretinal segmentation exist in the literature (e.g., [1]), such approaches have been two-dimensional in nature.

To address this need, we have previously reported an optimal 3-D graph search approach for the intraretinal layer segmentation of macular scans [2, 3. The approach is based on the optimal graph search method reported by $\mathrm{Wu}$ and Chen 4 and the extension to the multiple surface case by Li et al. [5]. In these approaches [4, 5], the surface segmentation problem is transformed into that of 


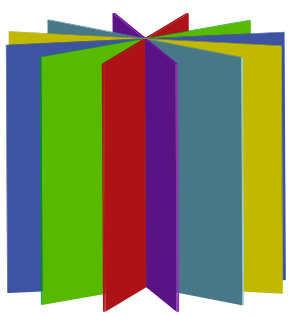

(a)

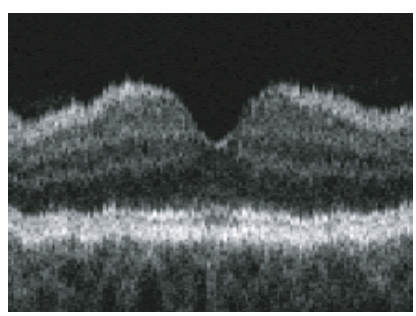

(b)

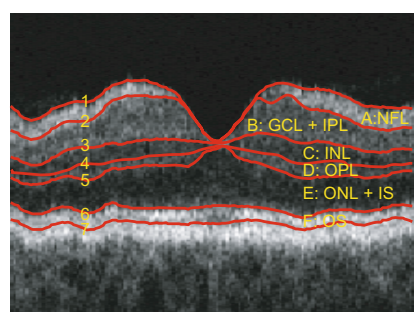

(c)

Fig. 1. Intraretinal layers on macular OCT images. (a) Schematic of six linear radial scans acquired during fast macular scanning protocol. Each color corresponds to one 2-D scan. (b) Example composite scan at one angular scan location. (c) Labeling of seven surfaces and corresponding six intraretinal layers on the 2-D composite scan.

finding a minimum-cost closed set in a constructed vertex-weighted geometric graph. Such a 3-D graph search method is well-suited for intraretinal layer segmentation for a variety of reasons. Perhaps the most important include 1) the ability to find an optimal set of surfaces with respect to a cost function in 3-D and 2) the ability to find multiple surfaces simultaneously. Note that even though the approach in [4,5] ultimately finds surfaces by finding a minimum-cost $s$ - $t$ cut in a constructed graph, it is fundamentally different than the "graph cut" methods of Boykov et al. (e.g., 6]).

However, in its original formulation, the 3-D optimal graph search method employs surface feasibility constraints that are constant in each direction. For example, the smoothness constraints for a particular surface $f(x, y)$ are represented by two parameters, $\Delta_{x}$ and $\Delta_{y}$, reflecting the allowed change in surface height when moving from one neighboring surface point to the next in the $x$-direction and $y$-direction, respectively. Similarly, the surface interaction constraints (reflecting the allowed minimum and maximum distances between surface pairs) are constant. More flexibility in constraining surfaces to particular shapes would be obtained if varying constraints were allowed. Such a change would especially be important for surfaces in which the needed constraints are expected to change based on location (e.g., the foveal region in OCT images).

In this work, we show how the optimal 3-D graph search may be extended to handle varying surface smoothness and surface interaction constraints. We then show how to learn such constraints from a training set and apply the method to the intraretinal layer segmentation of macular OCT images.

\section{Optimal 3-D Graph Search with Varying Constraints}

The optimal 3-D graph search approach is designed to solve what we will call the "multiple surface segmentation problem." In very general terms, the multiple surface segmentation problem can be thought of as an optimization problem with the goal being to find the set of surfaces with the minimum cost such that the found surface set is feasible. Thus, there are two major components 
to the problem specification: 1) the specification of the constraints to require surface set feasibility and 2) the formalization of the cost of a set of surfaces. The first step in the graph search approach is to construct a graph such that the minimum-cost closed set of the graph corresponds to the set of surfaces with the minimum cost. (A closed set is a subset of the vertices of a graph such that no directed edges leave the set.) This is done by 1) ensuring that there is a one-to-one correspondence between each closed set in the constructed graph and each feasible surface set and 2) ensuring that the cost of each closed set in the graph corresponds (within a constant) to the cost of a set of (feasible) surfaces. Then the actual minimum-cost closed set is found by finding minimum-cost $s$ - $t$ cut in a closely related graph 4,5. In this section, we describe the surface set feasibility constraints and costs in more detail, also briefly showing how each component is represented in the constructed graph.

\subsection{Surface Set Feasibility with Varying Constraints}

Consider a volumetric image $I(x, y, z)$ of size $X \times Y \times Z$. We focus on the case in which each surface of interest can be defined with a function $f(x, y)$ mapping $(x, y)$ pairs to $z$-values. Associated with each $(x, y)$ pair is a column of voxels in which only one of the voxels - the voxel at $(x, y, f(x, y))$ - intersects the surface. Each column also has a set of neighbors. We use a "4-neighbor" relationship in which the set of neighbors for the column associated with $(x, y)$ are the columns associated with $(x+1, y),(x-1, y),(x, y+1)$, and $(x, y-1)$.

In prior work, a single surface is considered feasible if the difference in $z$ values of neighboring surface points is less than or equal to a constant parameter ( $\Delta_{x}$ in $x$-direction, $\Delta_{y}$ in $y$-direction). For example, for neighboring columns $\{(x, y),(x+1, y)\}$ in the $x$-direction, this requires that

$$
-\Delta_{x} \leq f(x, y)-f(x+1, y) \leq \Delta_{x} .
$$

A similar constraint exists for neighbors in the $y$-direction.

In this work, we allow the smoothness constraints to vary as a function of the column neighborhood pair. For a given neighborhood pair $\left\{\left(x_{1}, y_{1}\right),\left(x_{2}, y_{2}\right)\right\}$, the constraint becomes:

$$
-\Delta_{\left\{\left(x_{1}, y_{1}\right),\left(x_{2}, y_{2}\right)\right\}}^{u} \leq f\left(x_{1}, y_{1}\right)-f\left(x_{2}, y_{2}\right) \leq \Delta_{\left\{\left(x_{1}, y_{1}\right),\left(x_{2}, y_{2}\right)\right\}}^{l},
$$

where $\Delta_{\left\{\left(x_{1}, y_{1}\right),\left(x_{2}, y_{2}\right)\right\}}^{u}$ reflects the maximum allowed increase in $z$-value when moving on a surface from column $\left(x_{1}, y_{1}\right)$ to column $\left(x_{2}, y_{2}\right)$ and $\Delta_{\left\{\left(x_{1}, y_{1}\right),\left(x_{2}, y_{2}\right)\right\}}^{l}$ reflects the maximum allowed decrease in $z$-value.

For a set of surfaces, additional constraints are added to model the desired relationships between the surfaces. For example, it may be known that one surface is always above another surface and that the distance between the surfaces is at least $\delta^{l}$ voxels, but no more than $\delta^{u}$ voxels (note the notational difference in $\delta$ used for surface interaction constraints and $\Delta$ used for smoothness constraints). Again, in prior work, these constraints were constant. In this work, we allow these constraints to be a function of $(x, y)$ so that in principle, a different interaction constraint can be used for each column. 


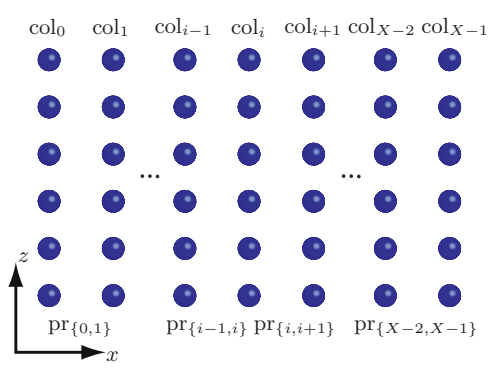

(a)

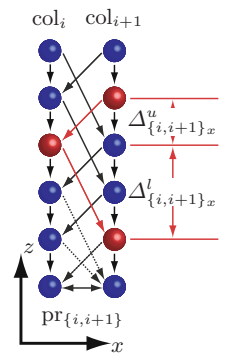

(b)

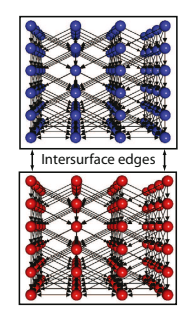

(c)

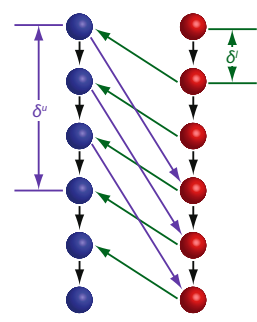

(d)

Fig. 2. Graph representation of feasibility constraints. (a-b) Surface smoothness constraints (shown only in $x$-direction using simplified notation). (c-d) Intersurface edges are added between the surface subgraphs to enforce the surface interaction constraints.

Graph representation. The structure of the constructed graph reflects the feasibility constraints. The graph is constructed in a similar manner as reported in [5] with the exception that the edges of the graph must take into account the varying constraints. First, one graph is created for each surface to be found. Then, intracolumn and intercolumn edges are added to enforce the surface smoothness constraints (Fig. 2(a-b)). Finally, the individual graphs are connected with intersurface edges to enforce the surface interaction constraints (Fig. 2(c-d)).

\subsection{Cost of a Feasible Surface Set}

We use the surface set cost formulation as presented in [3]. For completeness, we briefly review this formulation. Given a set of $n$ non-intersecting surfaces $\left\{f_{1}(x, y), f_{2}(x, y), \ldots, f_{n}(x, y)\right\}$, the surfaces naturally divide the volume into $n+1$ regions (Fig. 3). Assuming the surfaces are labeled in "increasing" order, the regions can be labeled $R_{0}, \ldots, R_{n}$, where $R_{i}$ reflects the region that lies between surface $i$ and surface $i+1$ (with region boundary cases $R_{0}$ and $R_{n}$ being defined as the region with lower $z$-values than surface 1 and the region with higher $z$ values than surface $n$, respectively). Each voxel can thus have $2 n+1$ real-valued costs associated with it: $n$ on-surface costs corresponding to the unlikeliness of belonging to each surface and $n+1$ in-region costs associated with the unlikeliness of belonging to each region. Let $c_{\mathrm{surf}_{i}}(x, y, z)$ represent the on-surface cost function associated with surface $i$ and $c_{\text {reg }_{i}}(x, y, z)$ represent the in-region cost function associated with region $i$. Then, the cost $C_{\left\{f_{1}(x, y), f_{2}(x, y), \ldots, f_{n}(x, y)\right\}}$ associated with the set of surfaces can be defined as

$$
C_{\left\{f_{1}(x, y), f_{2}(x, y), \ldots, f_{n}(x, y)\right\}}=\sum_{i=1}^{n} C_{f_{i}(x, y)}+\sum_{i=0}^{n} C_{R_{i}},
$$

where

$$
C_{f_{i}(x, y)}=\sum_{\left\{(x, y, z) \mid z=f_{i}(x, y)\right\}} c_{\operatorname{surf}_{i}}(x, y, z) \text { and } C_{R_{i}}=\sum_{(x, y, z) \in R_{i}} c_{\mathrm{reg}_{i}}(x, y, z) .
$$




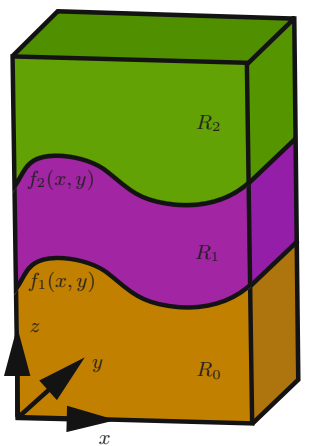

(a)

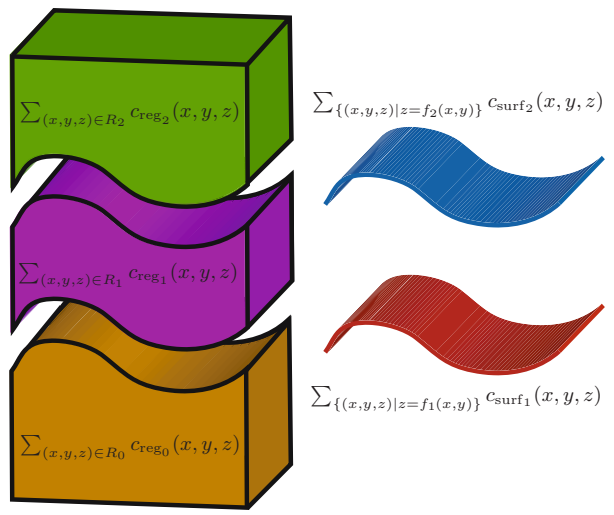

(b)

Fig. 3. Example schematic cost of two surfaces for the multiple surface segmentation problem. The two surfaces divide the volume into three regions.

Note that $C_{f_{i}(x, y)}$ reflects the cost associated with voxels on surface $i$ and $C_{R_{i}}$ reflects the cost associated with voxels belonging to region $i$.

Graph representation. The cost of each vertex in the graph is set such that the cost of each closed set corresponds to the cost (within a constant) of the set of surfaces. (The cost of a closed set is the summation of the costs of all the vertices.) The weight $w_{i}(x, y, z)$ of each vertex $(i=1,2, \ldots, n)$ can be defined as the summation of a term related to the on-surface costs $\left(w_{\text {on- }} \operatorname{surf}_{i}(x, y, z)\right)$ and a term related to the in-region costs $\left(w_{\text {in- }-\operatorname{reg}_{i}}(x, y, z)\right)$ :

$$
w_{i}(x, y, z)=w_{\mathrm{on}-\operatorname{surf}_{i}}(x, y, z)+w_{\text {in }-\operatorname{reg}_{i}}(x, y, z) .
$$

For on-surface costs, the cost of each vertex is assigned the on-surface cost of the corresponding voxel minus the on-surface cost of the voxel below it [4, 5]:

$$
w_{\text {on- } \operatorname{surf}_{i}}(x, y, z)=\left\{\begin{array}{ll}
c_{\operatorname{surf}_{i}}(x, y, z) & \text { if } z=0 \\
c_{\operatorname{surf}_{i}}(x, y, z)-c_{\operatorname{surf}_{i}}(x, y, z-1) & \text { otherwise }
\end{array} .\right.
$$

For in-region costs, the cost of each vertex is assigned the in-region cost of the region below the surface associated with the vertex minus the in-region cost of the region above the surface associated with the vertex:

$$
w_{\text {in }-\operatorname{reg}_{i}}(x, y, z)=c_{\operatorname{reg}_{i-1}}(x, y, z)-c_{\operatorname{reg}_{i}}(x, y, z) .
$$

\section{Application to Intraretinal Layer Segmentation}

\subsection{Segmentation Overview}

To increase the signal to noise ratio on the macular OCT images, up to six raw macular series are first aligned and registered together using the methods 
described in [2]. This results in a composite 3-D scan for each eye. Then, for each composite 3-D OCT image, the seven surfaces are found in two groups using the optimal graph search approach: first, surfaces 1, 6, and 7 are found simultaneously. Then surfaces 2, 3, 4, and 5 are simultaneously determined. The cost functions are the same as we previously reported in [3]. Basically, the surfaces in the first group use on-surface costs based on image gradient and localized regional information, while the surfaces in the second group use inregion costs based on fuzzy membership values determined from image intensity.

\subsection{Learning the Constraints}

For each subject, the smoothness and surface interaction constraints are learned in a leave-one-subject-out fashion. For purposes of learning the varying smoothness constraints for each surface, it may be easiest to think of each pair of neighboring columns $\left\{\left(x_{1}, y_{1}\right),\left(x_{2}, y_{2}\right)\right\}$ as having its own constraint that needs to be learned separately. The basic idea is use manual tracings in the training set to determine the mean and standard deviation of how the $z$-value changes when moving from column $\left(x_{1}, y_{1}\right)$ to column $\left(x_{2}, y_{2}\right)$. Let $\bar{d}$ reflect the mean deviation (i.e., the mean of $f\left(x_{1}, y_{1}\right)-f\left(x_{2}, y_{2}\right)$ from the reference standard) and $\sigma$ reflect the standard deviation. Then, to allow for $99 \%$ of the expected changes in $z$ value when moving from column $\left(x_{1}, y_{1}\right)$ to column $\left(x_{2}, y_{2}\right)$ (assuming a normal distribution), the two parameters of the smoothness constraint, $\Delta_{\left\{\left(x_{1}, y_{1}\right),\left(x_{2}, y_{2}\right)\right\}}^{l}$ and $\Delta_{\left\{\left(x_{1}, y_{1}\right),\left(x_{2}, y_{2}\right)\right\}}^{u}$ can be set as follows:

$$
\Delta_{\left\{\left(x_{1}, y_{1}\right),\left(x_{2}, y_{2}\right)\right\}}^{l}=\bar{d}+2.6 * \sigma \quad \text { and } \quad \Delta_{\left\{\left(x_{1}, y_{1}\right),\left(x_{2}, y_{2}\right)\right\}}^{u}=-(\bar{d}-2.6 * \sigma) .
$$

In this work, varying surface interaction constraints are used only in the simultaneous segmentation of the interior surfaces 2, 3, 4 and 5 . The varying constraint is computed for the following surfaces pairings: 1-2, 2-3, 3-4, 4-5, and 5 -6. For each surface pairing, the minimum distance between the surfaces at each column is set as the mean leave-one-out thickness minus 2.6 times the standard deviation (but truncated so as not to go below 0). Similarly, the maximum distance between the surfaces at each column is set as the mean leave-one-out thickness plus 2.6 times the standard deviation.

\subsection{Experimental Methods}

The intraretinal layer segmentation algorithm using varying constraints is applied to the composite 3 -D images from fast macular scans from 12 subjects (24 eyes) with unilateral chronic anterior ischemic optic neuropathy. In addition, for comparison purposes, the algorithm is also applied to the same images using the constant smoothness and surface interaction constraints reported in [3]. Each composite 3 -D image is comprised of six scans, each $128 \times 1024$ pixels. The physical width and height of the 2-D raw scans (and thus also the composite scans) is $6 \mathrm{~mm} \times 2 \mathrm{~mm}$, resulting in a pixel size of approximately $50 \mu \mathrm{m}$ (laterally) $\times 2 \mu \mathrm{m}$ (axially). One raw scan from each eye is independently traced by two 
human experts with the average of the two tracings being used as the reference standard. Borders that are not considered visible are not traced. The algorithmic result on the corresponding composite $2-\mathrm{D}$ scan is converted into the coordinate system of the raw scan (inversely transforming the alignment/registration) and the mean unsigned border positioning errors for each border are computed. The unsigned border positioning errors are also computed using one observer as a reference standard for the other.

\section{Results}

The computed unsigned border positioning errors are summarized in Table 1 . The smallest errors are consistently obtained for the variation of the algorithm using both varying smoothness and varying surface interaction constraints. For example, the overall (all surfaces combined) mean unsigned border positioning error is $7.3 \pm 3.7 \mu \mathrm{m}$ using both varying smoothness and varying interaction constraints and $8.3 \pm 4.9 \mu \mathrm{m}$ using constant constraints. These results compare favorably with the overall mean inter-observer variability of $8.2 \pm 3.5 \mu \mathrm{m}$ and the reported 9-10 $\mu \mathrm{m}$ axial resolution of the scanner system. The systematic improvement caused by introducing the varying constraints is at the $10 \%$ level overall. Even more noticeable differences occur in cases in which image information is locally ambiguous. Fig. 4 shows an example improvement.

Table 1. Summary of mean unsigned border positioning errors ${ }^{\dagger}$

\begin{tabular}{cccc}
\hline & & \multicolumn{2}{c}{ Algorithm vs. Avg. Observer } \\
\cline { 3 - 4 } Border & Obs. 1 vs. Obs. 2 & Varying S \& I & Constant S \& I \\
\hline 1 & $5.9 \pm 1.2$ & $4.6 \pm 1.6$ & $5.1 \pm 2.3$ \\
$2^{\ddagger}$ & $6.3 \pm 1.0$ & $10.9 \pm 4.3$ & $11.2 \pm 5.0$ \\
$3^{\ddagger}$ & $9.1 \pm 3.7$ & $8.9 \pm 4.4$ & $10.2 \pm 4.9$ \\
$4^{\ddagger}$ & $7.6 \pm 2.0$ & $8.6 \pm 1.9$ & $12.0 \pm 4.5$ \\
5 & $9.4 \pm 3.9$ & $8.3 \pm 2.9$ & $9.7 \pm 5.4$ \\
6 & $7.6 \pm 2.4$ & $3.0 \pm 1.0$ & $3.6 \pm 2.5$ \\
7 & $11.4 \pm 4.7$ & $7.6 \pm 2.2$ & $7.8 \pm 2.2$ \\
\hline Overall & $8.2 \pm 3.5$ & $7.3 \pm 3.7$ & $8.3 \pm 4.9$ \\
\hline${ }^{\dagger}$ Mean \pm SD in $\mu \mathrm{m}$ for 24 scans. \\
${ }^{\ddagger}$ Errors were not computed for those scans in which boundary was \\
determined to not be visible by at least one observer.
\end{tabular}

\section{Discussion and Conclusion}

We have presented a method for the incorporation of varying constraints to the optimal 3-D graph search approach. In applying varying constraints to the intraretinal layer segmentation of optical coherence tomography images we 


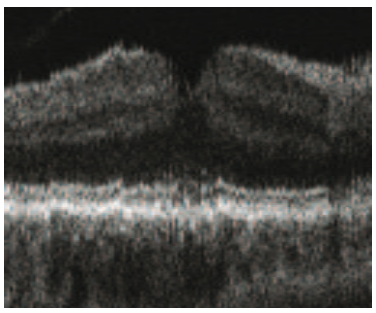

(a) Original

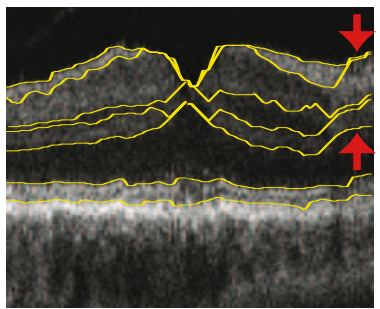

(b) Constant S \& I

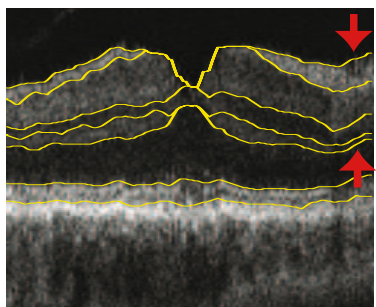

(c) Varying S \& I

Fig. 4. Example improvement in segmentation result by using varying constraints

obtained a quantitative improvement in the results. Qualitatively, the varying constraints seemed to help the most in cases where the image information was less certain. In these cases, the constraints helped to ensure that a segmentation with a more feasible shape would be obtained. Thus, in addition to improving the cost function, incorporating varying constraints is another means of improving the segmentation for applications using the optimal 3-D graph search.

\section{References}

1. Cabrera Fernández, D., Salinas, H.M., Puliafito, C.A.: Automated detection of retinal layer structures on optical coherence tomography images. Opt. Express 13(25), 10200-10216 (2005)

2. Haeker, M., Sonka, M., Kardon, R., Shah, V.A., Wu, X., Abràmoff, M.D.: Automated segmentation of intraretinal layers from macular optical coherence tomography images (651214). In: Pluim, J.P.W., Reinhardt, J.M. (eds.) Proc. of SPIE Medical Imaging 2007: Image Processing. SPIE, vol. 6512, 651214 (2007)

3. Haeker, M., Wu, X., Abràmoff, M.D., Kardon, R., Sonka, M.: Incorporation of regional information in optimal 3-D graph search with application for intraretinal layer segmentation of optical coherence tomography images. In: Karssemeijer, N., Lelieveldt, B. (eds.) IPMI 2007. LNCS, vol. 4584, pp. 607-618. Springer, Heidelberg (2007)

4. Wu, X., Chen, D.Z.: Optimal net surface problems wih applications. In: Widmayer, P., Triguero, F., Morales, R., Hennessy, M., Eidenbenz, S., Conejo, R. (eds.) ICALP 2002. LNCS, vol. 2380, pp. 1029-1042. Springer, Heidelberg (2002)

5. Li, K., Wu, X., Chen, D.Z., Sonka, M.: Optimal surface segmentation in volumetric images - a graph-theoretic approach. IEEE Trans. Pattern Anal. Machine Intell. 28(1), 119-134 (2006)

6. Boykov, Y., Jolly, M.P.: Interactive organ segmentation using graph cuts. In: Delp, S.L., DiGoia, A.M., Jaramaz, B. (eds.) MICCAI 2000. LNCS, vol. 1935, pp. 276-286. Springer, Heidelberg (2000) 